\title{
EXPERIMENTAL AND FINITE ELEMENT ANALYSIS ON THE RESPONSE AND FAILURE OF LOCAL SHARP-DENTED 6061-T6 ALUMINUM ALLOY TUBES SUBJECTED TO CYCLIC BENDING
}

\author{
KUO-LONG LEE ${ }^{1}$, YI-FU CHIU ${ }^{2} \&$ WEN-FUNG PAN ${ }^{2}$ \\ ${ }^{1}$ Department of Innovative Design and Entrepreneurship Management, Far East University, Taiwan \\ ${ }^{2}$ Department of Engineering Science, National Cheng Kung University, Taiwan
}

\begin{abstract}
In this paper, the response and failure of local sharp-dented 6061-T6 aluminum alloy tubes with different dent depths of $0.3,0.6,0.9$ and $1.2 \mathrm{~mm}$ subjected to cyclic bending was experimentally investigated. The tube-bending machine and curvature-ovalization measurement apparatus were used for conducting the curvature-controlled cyclic bending experiments. It was observed that the momentcurvature relationship showed an almost steady loop from the beginning of the first cycle. However, the ovalization-curvature relationship exhibited an increasing and ratcheting way with the number of the bending cycles. In addition, a deeper dent depth of a tube led to a more severe asymmetrical trend of the ovalization-curvature relationship. It has been demonstrated that the dent depth has small influence on the moment-curvature relationship. But, it has a strong influence on the ovalization-curvature relationship. Next, using proper stress-strain relationship, model, mesh, boundary conditions and loading conditions, the finite element ANSYS was used to describe the moment-curvature and ovalization-curvature relationships. Because the failure type was the fatigue fracture, the ANSYS was used to calculate the maximum and minimum stresses for each cyclic bending case. Finally, a simple fatigue model was proposed for predicting the stress amplitude-number of bending cycles required to produce failure relationship. It was found that the experimental and analytical data agreed quite well.
\end{abstract}

Keywords: 6061-T6 aluminum alloy tubes, local sharp dent, cyclic bending, moment, curvature, ovalization, finite element ANSYS analysis.

\section{INTRODUCTION}

It is well known that the bending of circular tubes causes cross section ovalization. The definition of ovalization is the change in the outer diameter divided by the original outer diameter. This ovalization increases slowly during reverse bending and continuous cyclic bending, and in turn, results in the degradation of the circular tube, which buckles or fractures when the ovalization reaches a critical value. Circular tubes are severely damaged during buckling and fracturing and cannot bear any load, which ultimately results in obstruction and leakage of the material being transported. As such, a complete understanding of the response and failure of circular tubes under cyclic bending is essential for industrial applications.

In 1998, Pan et al. [1] designed and set up a new measurement apparatus. It was used with the cyclic bending machine to study various kinds of tubes under different cyclic bending conditions. For instance, Pan and Fan [2] studied the effect of the prior curvature-rate at the preloading stage on subsequent creep (moment is kept constant for a period of time) or relaxation (curvature is kept constant for a period of time) behavior, Pan and Her [3] investigated the response and stability of SUS304 stainless steel tubes that were subjected to cyclic bending with different curvature-rates, Lee et al. [4] studied the influence of the diameter-to-thickness ratio on the response and stability of circular tubes that were subjected 
to symmetrical cyclic bending, Lee et al. [5] experimentally explored the effect of the diameter-to-thickness ratio and curvature rate on the response and stability of circular tubes subjected to cyclic bending, and Chang and Pan [6] discussed the buckling life estimation of circular tubes subjected to cyclic bending.

In practical industrial applications, tubes are under the hostile environment, so the material in the environment may corrode the tube surface and produce notches. Additionally, a tube in the working condition often involves some notches. The mechanical behavior and buckling failure of a notched tube differs from that of a tube with a smooth surface. In 2010, Lee et al. [7] studied the variation in ovalization of sharp-notched circular tubes subjected to cyclic bending. Lee [8] investigated the mechanical behavior and buckling failure of sharp-notched circular tubes under cyclic bending. Lee et al. [9] experimentally discussed the viscoplastic response and collapse of sharp-notched circular tubes subjected to cyclic bending. Later, Lee et al. [10] investigated the response of SUS304 stainless steel tubes subjected to pure bending creep and pure bending relaxation.

In 2012, Limam et al. [11] experimentally and analytically inspected the collapse curvature of locally-dented tubes subjected to pure bending with internal pressure. In their experiments, 321 stainless steel tubes with outer diameters of 1.5 in. and diameter-tothickness ratio of 52 were tested. The dented tubes were internally pressurized and then bent to collapse. They found that such defects decreased the bending rigidity quite significantly. In addition, finite element models were employed to simulate the denting on tubes, internal pressurization, and bending to collapse.

In this paper, local sharp-dented 6061-T6 aluminum alloy tubes with different dent depths under cyclic bending were experimentally studied. Related experimental tests were conducted using a tube-bending machine and curvature-ovalization measurement apparatus. The bending moment, curvature, and ovalization were measured with sensors at the testing facility. The number of bending cycles required to produce failure was also recorded.

\section{EXPERIMENTS}

By using a tube-bending device and a curvature-ovalization measurement apparatus, local sharp-dented 6061-T6 aluminum alloy tubes with different dent depths and subjected to cyclic bending were studied. The experimental device, materials, specimens, and test procedures are detailed below.

\subsection{Experimental device}

The experiments were performed in a specially-built testing facility, shown schematically in Fig. 1. This was designed to perform bending, reverse, and cyclic tests. A detailed explanation of the experimental facility can be found in many papers (Pan and Fan [2], Pan and Her [3], Lee et al. [4]). Fig. 2 schematically shows the light-weight instrument designed by Pan et al. [1] for measuring the curvature and ovalization of a tube. The angle variation during cyclic bending can be measured by two side-inclinometers in the instrument. The tube curvature can be obtained by a simple calculation, i.e., an extended version of the calculation described by Pan et al. [1].

\subsection{Materials and specimen}

6061-T6 aluminum alloy tubes were used for the experiment. The chemical composition of the tested material is given in Table 1 . The mechanical properties were as follows: ultimate stress of $258 \mathrm{MPa}$, yield stress of $166 \mathrm{MPa}$, and percent elongation of $23 \%$. 


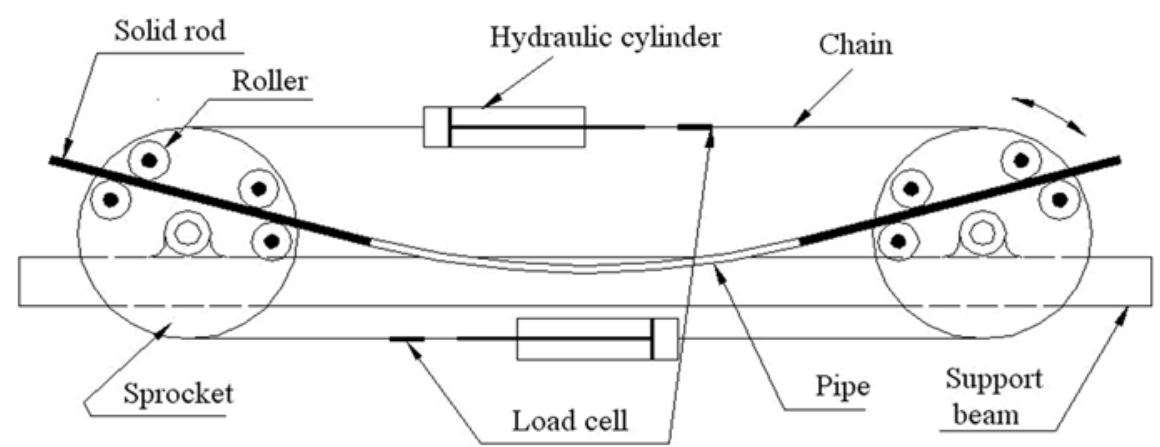

Figure 1: Schematic drawing of the tube bending machine.

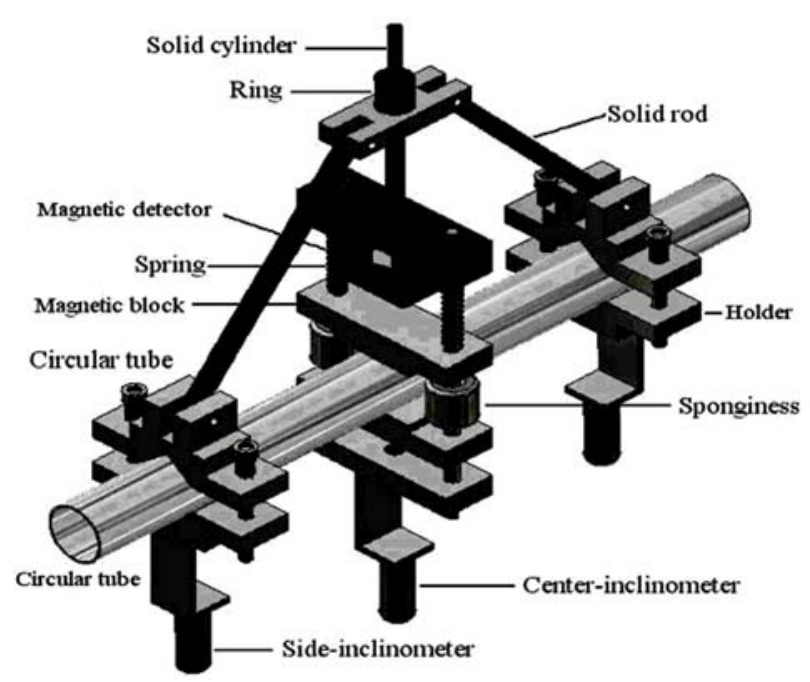

Figure 2: Schematic drawing of the curvature-ovalization measurement apparatus.

Table 1: Chemical composition of 6061-T6 aluminum alloy (weight \%).

\begin{tabular}{|llllllllll|}
\hline Chemical composition & $\mathrm{Al}$ & $\mathrm{Mg}$ & $\mathrm{Si}$ & $\mathrm{Fe}$ & $\mathrm{Zn}$ & $\mathrm{Mn}$ & $\mathrm{Cr}$ & $\mathrm{Ti}$ & $\mathrm{Ni}$ \\
\hline Proportion (\%) & 98.096 & 0.937 & 0.535 & 0.139 & 0.0983 & 0.022 & 0.022 & 0.012 & 0.005 \\
\hline
\end{tabular}

The outer surfaces of the original tubes with outer diameter $D_{\mathrm{o}}$ of $33.0 \mathrm{~mm}$ and wall thickness $t$ of $2.0 \mathrm{~mm}$ were processed to introduce the expected dents. Figs 3(a) and 3(b) show a picture and a schematic drawing of the dent production, respectively. The upper mold made contact with the tube surface and exerted a pressure F to create the dent. In this study, four different dent depths (a) were considered: 0.3, 0.6, 0.9, and $1.2 \mathrm{~mm}$. 


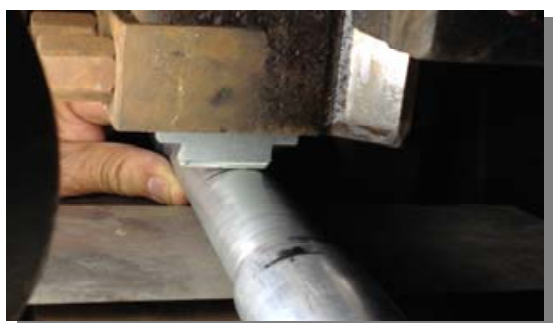

(a)

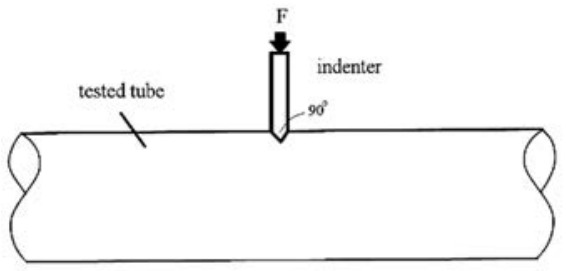

(b)

Figure 3: (a) Picture of processing a sharp dent on the 6061-T6 aluminum alloy tube; (b) Schematic drawing of processing a sharp dent on the 6061-T6 aluminum alloy tube.

\subsection{Test procedures}

The bending experiments were performed under curvature-controlled conditions. The curvature rate for the cyclic bending test was $0.03 \mathrm{~m}^{-1} / \mathrm{sec}$. Two load cells installed in the testing facility (Fig. 1) were used to measure the bending moment. The instrument in Fig. 2 was used to measure the curvature and ovalization. The number of bending cycles required to produce failure was also recorded.

\section{FINITE ELEMENT ANSYS ANALYSIS}

In this study, the response of local sharp-dented circular tubes subjected to cyclic bending was also analyzed numerically using the finite element code ANSYS. The response is the correlation among the moment, curvature, and ovalization. The elastic-plastic stress-strain response, model, mesh, boundary conditions, and loading conditions are explained below.

\subsection{Elastic-plastic stress-strain curves}

Fig. 4 shows the tested and ANSYS-constructed uniaxial stress $(\sigma)$-strain $(\varepsilon)$ curves for the 6061-T6 aluminum alloy. The kinematic hardening rule was used as the hardening rule for cyclic loading.

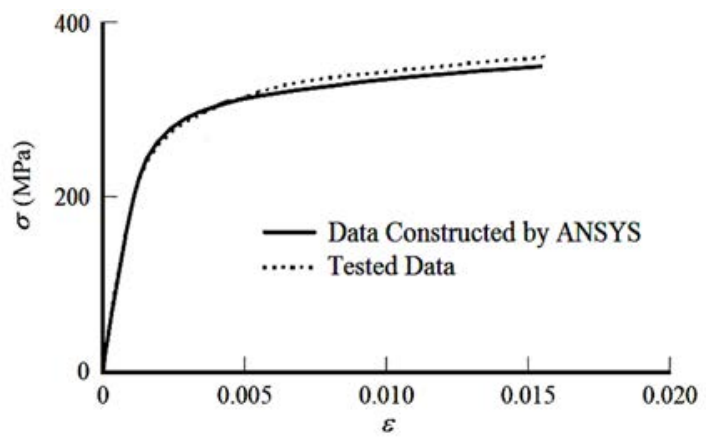

Figure 4: Tested and ANSYS-constructed data of the uniaxial stress $(\sigma)$-strain $(\varepsilon)$ curve for 6061-T6 aluminum alloy. 


\subsection{Model}

The model consisted of three parts: the sharp-dented tube, the indenter, and the solid rod. The size and geometric shape of the sharp-dented tube are stated in a previous section and the schematic drawings of the indenter and solid rod are shown in Figs 5(a) and 5(b), respectively. It can be seen in Fig. 5(a) that production of a sharp dent includes the indenter and a fixed plate. The indenter exerts a pressure on the tube related to the desired depth of the dent. The shape and size of the dents are discussed in a previous section.

\subsection{Mesh}

Due to the three-dimensional geometry and elastic-plastic deformation of the tube, the SOLID 185 element was used for relative analysis. This is a tetrahedral element built in ANSYS and is suitable for analyzing plastic or large deformations. In particular, the element can adequately analyze shell components under bending. Due to the right and left symmetry, only half of the tube model was constructed. Fig. 6(a) shows the mesh constructed by ANSYS for the indenter and half tube, and Fig. 6(b) shows the mesh constructed by ANSYS for the half tube.

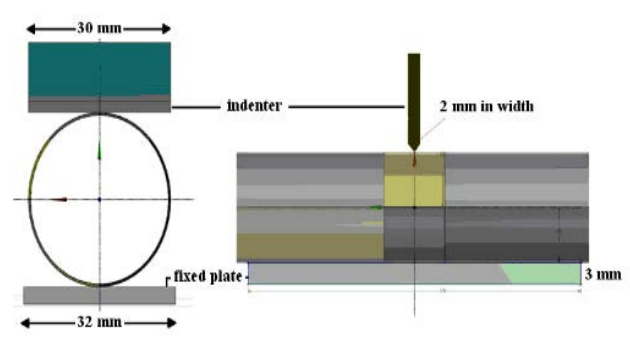

(a)

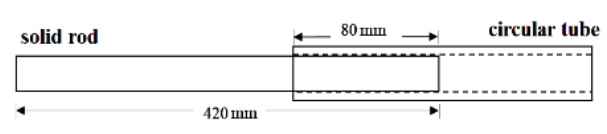

(b)

Figure 5: (a) Schematic drawing of the indenter; (b) Schematic drawing of the solid rod.

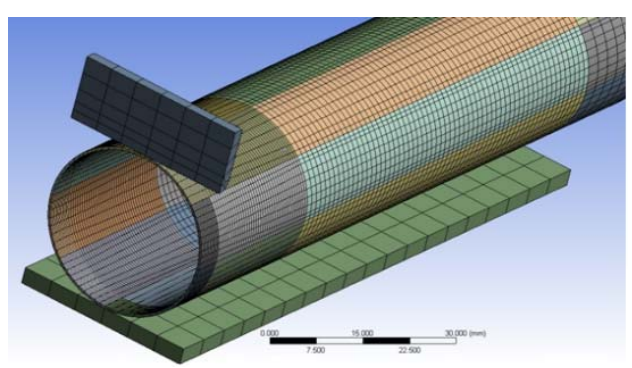

(a)

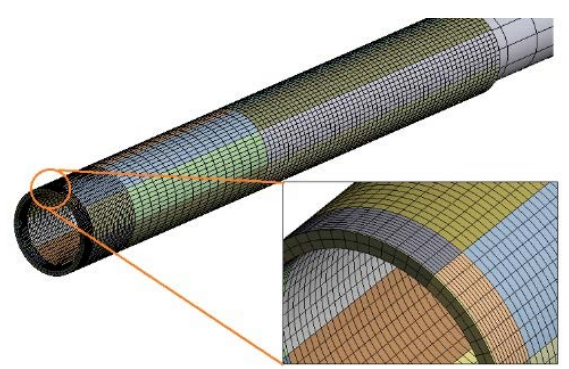

(b)

Figure 6: (a) Mesh constructed by ANSYS for the indenter; (b) Mesh constructed by ANSYS for half tube. 


\subsection{Boundary conditions}

When producing a sharp dent on the tube, the indenter moved in the y-direction only. Therefore, friction supports were set to prevent any displacement in the $\mathrm{x}$ - or z-directions, as shown in Fig. 7(a). The indenter was set to exert pressure on the tube to produce a dent and then to reset back to its original position. The contact between the indenter and tube was set to be frictionless, as shown in Fig. 7(a). The plate was fixed, thus, a fixed support was set on the plate as shown in Fig. 7(b). As there was no related displacement between the tube and the fixed plate, the bonded contact between them was used, as shown in Fig. 7(b).

\subsection{Loading conditions}

When producing a dent, the indenter was set to move downward to create a desired dent depth, then travel back to its original position. Fig. 8(a) shows the loading conditions of the indenter as constructed by ANSYS, and Fig. 8(b) shows the loading condition constructed by ANSYS on the basis of the tube-bending device. As shown, remote displacement of the solid rod in the $\mathrm{z}$-direction was unrestricted, hence, the rotations in the $\mathrm{x}$ - and $\mathrm{y}$-directions were set to zero.

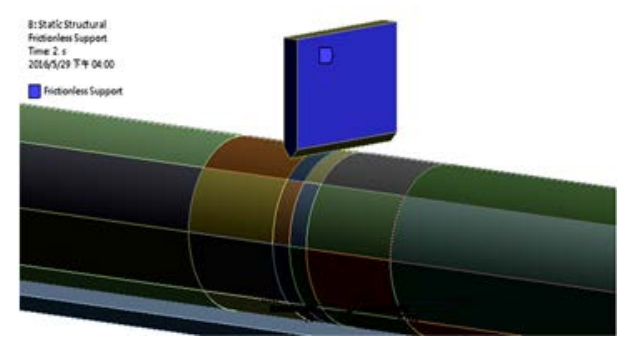

(a)

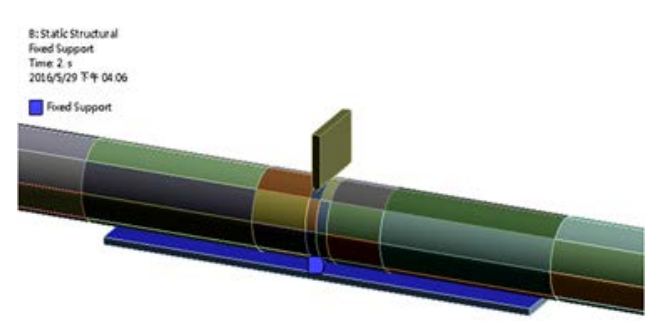

(b)

Figure 7: (a) Boundary conditions for the indenter; (b) Boundary conditions for the contact between the indenter and tube.

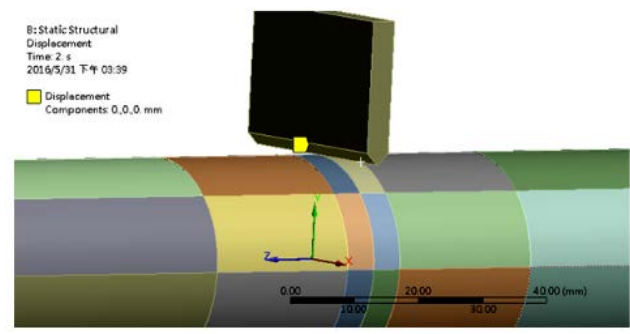

(a)

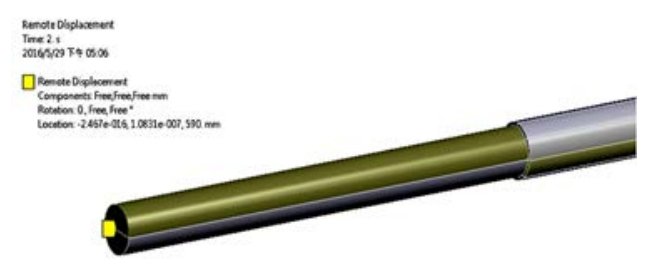

(b)

Figure 8: (a) Loading conditions for the indenter; (b) Loading conditions for the solid rod. 
Fig. 9 depicts a tube subjected to pure bending. The rotating angle $\theta$ was used as input data for curvature-controlled cyclic bending. The curvature $\kappa$ is

$$
\kappa=1 / \rho=2 \theta / L_{0},
$$

where $\rho$ is the radius of curvature and $L_{\mathrm{o}}$ is the original tube length.

\section{EXPERIMENTAL AND SIMULATED RESULTS}

\subsection{Mechanical response}

A typical set of experimental relationship between moment $(M)$ and curvature $(\kappa)$ for local sharp-dented 6061-T6 aluminum alloy tubes with $a=0.0 \mathrm{~mm}$ subjected to cyclic bending is shown in Fig. 10(a). Note that $a=0.0 \mathrm{~mm}$ represents the smooth tube. The tubes were cycled between curvatures of $\pm 0.4 \mathrm{~m}^{-1}$. It can be observed that the $M-\kappa$ response is a stable hysteresis loop from the first bending cycle. Since the dent is small and local, the dent depth has almost no influence on the $M-\kappa$ curve. Therefore, the $M-\kappa$ curves for different $a$ are not shown in this paper. Fig. 10(b) shows the corresponding simulated $M-\kappa$ curve by ANSYS.

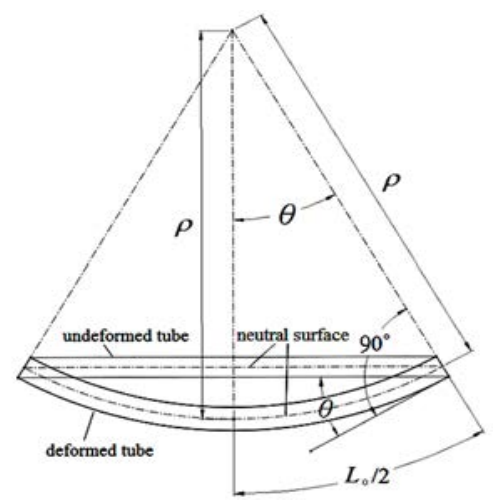

Figure 9: Relationship between rotating angle $\theta$ and curvature $\kappa$ for a tube under pure bending.

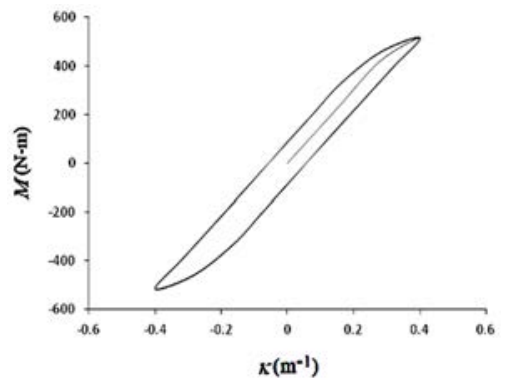

(a)

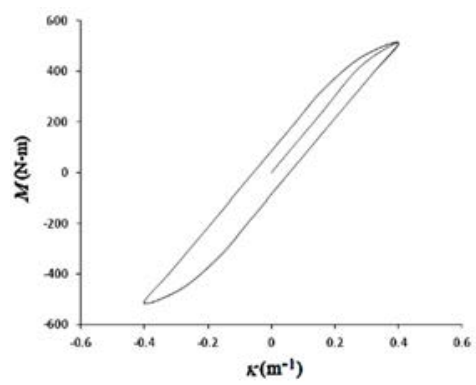

(b)

Figure 10: (a) Experimental; (b) ANSYS simulated moment $(M)$-curvature $(\kappa)$ curves for local sharp-dented 6061-T6 aluminum alloy tubes with $a=0.0 \mathrm{~mm}$ under cyclic bending. 
The ovalization $\left(\Delta D_{\mathrm{o}} / D_{\mathrm{o}}\right)$-curvature $(\kappa)$ relationships for $a=0.0,0.3,0.6,0.9$, and $1.2 \mathrm{~mm}$ are shown in Figs 11(a)-(e), respectively. The tubes were also cycled between curvatures of $\pm 0.4 \mathrm{~m}^{-1}$. It is noted that the $\Delta D_{\mathrm{o}} / D_{0}-\kappa$ curves exhibit a ratcheting trend and increase with the number of bending cycles. A larger $a$ results in a more asymmetrical look to the $\Delta D_{\mathrm{o}} / D_{0}-\kappa / \kappa_{0}$ curve. Moreover, a larger $a$ leads to a larger ovalization. Figs 12(a)-(e) depict the corresponding ANSYS simulated $\Delta D_{\mathrm{o}} / D_{\mathrm{o}}-\kappa$ curves for Figs $11(\mathrm{a})-(\mathrm{d})$, respectively.

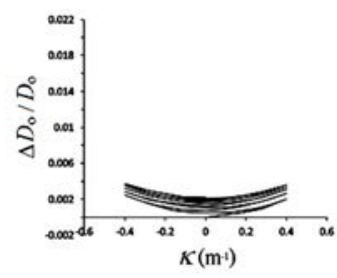

(a)

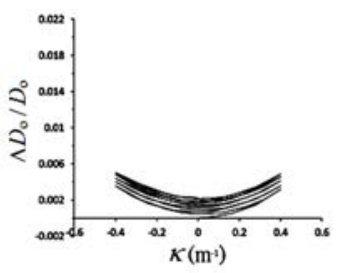

(b)

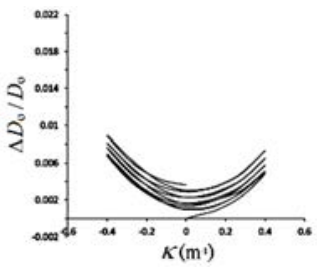

(c)

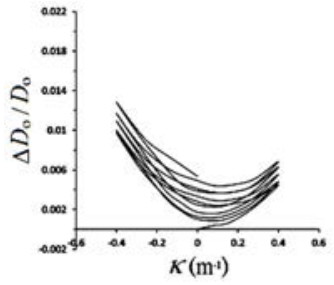

(d)

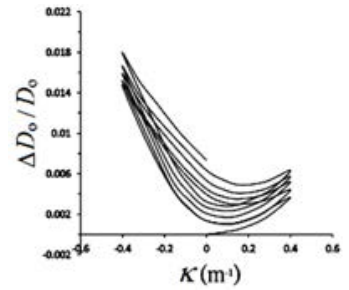

(e)

Figure 11: Experimental ovalization $\left(\Delta D_{0} / D_{0}\right)$-curvature $(\kappa)$ curves for local sharp-dented 6061-T6 aluminum alloy tubes with $a=$ (a) 0.0 , (b) 0.3 , (c) 0.6 , (d) 0.9 and (e) $1.2 \mathrm{~mm}$ under cyclic bending.

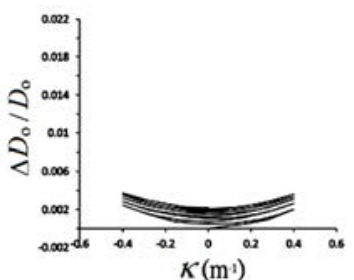

(a)

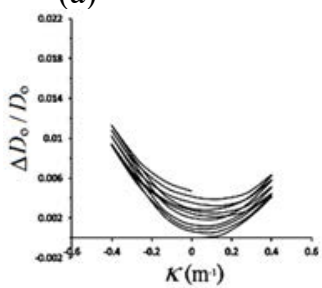

(d)

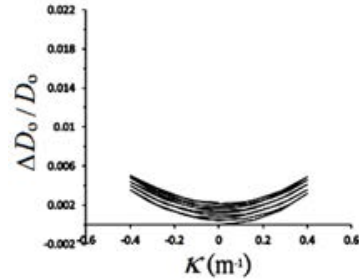

(b)

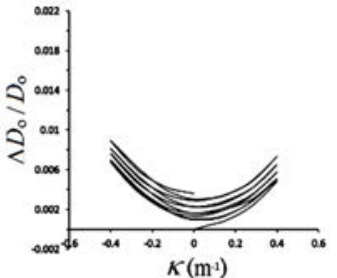

(c)

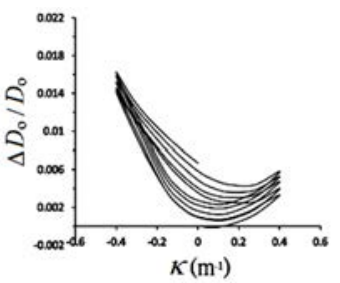

(e)

Figure 12: ANSYS simulated ovalization $\left(\Delta D_{\mathrm{o}} / D_{\mathrm{o}}\right)$-curvature $(\kappa)$ curves for local sharpdented 6061-T6 aluminum alloy tubes with $a=$ (a) 0.0 ; (b) 0.3 ; (c) 0.6 ; (d) 0.9 ; and (e) $1.2 \mathrm{~mm}$ under cyclic bending. 


\subsection{Fatigue failure}

Fig. 13 shows a picture of the failure for local sharp-dented 6061-T6 aluminum alloy circular tubes with $a=0.0,0.3,0.6,0.9$ and $1.2 \mathrm{~mm}$ under cyclic bending. The red circles denote the initiation of the crack. It was found that the crack initiated at the one or both sides of the sharp dent. In addition, once the crack initiation was observed, the tube broke rapidly.

Table 2 shows the maximum and minimum stresses for local sharp-dented 6061-T6 aluminum alloy circular tubes subjected to cyclic bending. The magnitudes of stress were

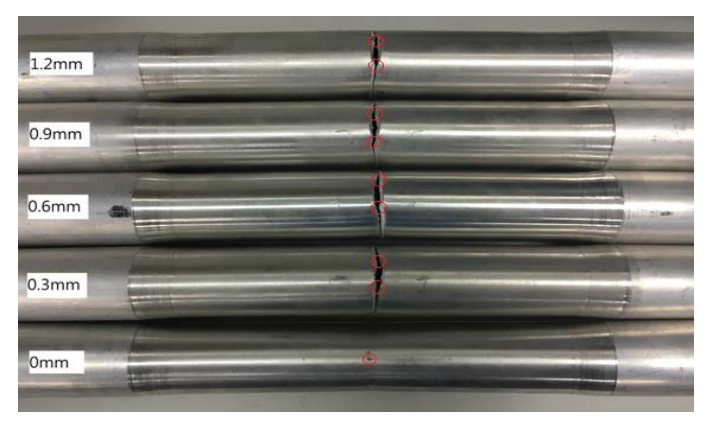

Figure 13: Picture of the fatigue failure for local sharp-dented 6061-T6 aluminum alloy circular tubes with different $a$ under cyclic bending.

Table 2: $\quad$ ANSYS determined the maximum and minimum stresses for local sharp-dented 6061-T6 aluminum alloy circular tubes subjected to cyclic bending.

\begin{tabular}{|c|c|c|c|}
\hline $\begin{array}{l}\text { Dent depth } \\
(\mathrm{mm})\end{array}$ & $\begin{array}{c}\text { Control curvature } \\
\left(\mathrm{m}^{-1}\right)\end{array}$ & $\begin{array}{c}\text { Maximum stress } \\
(\mathrm{MPa})\end{array}$ & $\begin{array}{c}\text { Minimum stress } \\
(\mathrm{MPa})\end{array}$ \\
\hline 0.0 & \pm 1.1 & 184.34 & -184.14 \\
\hline 0.0 & \pm 0.9 & 150.03 & -149.88 \\
\hline 0.0 & \pm 0.7 & 131.98 & -131.66 \\
\hline 0.0 & \pm 0.6 & 130.34 & -129.99 \\
\hline 0.0 & \pm 0.5 & 128.83 & -128.27 \\
\hline 0.3 & \pm 0.7 & 164.08 & -164.02 \\
\hline 0.3 & \pm 0.6 & 159.24 & -159.03 \\
\hline 0.3 & \pm 0.5 & 154.98 & -154.66 \\
\hline 0.3 & \pm 0.4 & 151.96 & -151.62 \\
\hline 0.3 & \pm 0.35 & 149.55 & -149.21 \\
\hline 0.6 & \pm 0.5 & 189.46 & -189.40 \\
\hline 0.6 & \pm 0.4 & 172.81 & -172.41 \\
\hline 0.6 & \pm 0.35 & 169.70 & -169.15 \\
\hline 0.6 & \pm 0.63 & 166.23 & -166.13 \\
\hline 0.6 & \pm 0.25 & 161.33 & -161.12 \\
\hline 0.9 & \pm 0.5 & 225.98 & -225.55 \\
\hline 0.9 & \pm 0.4 & 215.51 & -215.35 \\
\hline 0.9 & \pm 0.35 & 199.47 & -199.33 \\
\hline 0.9 & \pm 0.3 & 196.05 & -195.88 \\
\hline 0.9 & \pm 0.25 & 190.27 & -189.98 \\
\hline 1.2 & \pm 0.45 & 241.37 & -241.22 \\
\hline 1.2 & \pm 0.35 & 227.40 & -227.11 \\
\hline 1.2 & \pm 0.3 & 222.58 & -222.32 \\
\hline 1.2 & \pm 0.25 & 202.25 & -202.17 \\
\hline 1.2 & \pm 0.2 & 188.01 & -187.65 \\
\hline
\end{tabular}


determined by ANSYS discussed in Section 3. It was observed that for a given dent depth, a tube with a higher value of control curvature led to higher values of maximum and minimum stresses. In addition, for a given control curvature, a tube with a higher value of dent depth led to higher values of maximum and minimum stresses. Due to the symmetrical curvaturecontrolled cyclic bending (the maximum control curvature equals to the minimum control curvature) and a local and small dent, the amounts of maximum and minimum stresses were almost the same for each loading case. Therefore, the mean stress effect did not consider in the fatigue analysis.

Fig. 14 shows the experimental data of the stress amplitude $\left(\sigma_{\mathrm{a}}\right)$ versus the number of bending cycles required to produce failure $\left(2 N_{\mathrm{f}}\right)$ in solid black circles for local sharp-dented 6061-T6 aluminum alloy circular tubes with different dent depths subjected to cyclic bending on a log-log scale. Note that $\sigma_{\mathrm{a}}=$ (maximum stress - minimum stress) / 2. It was observed that the experimental data were almost in a straight line. Therefore, a simple fatigue model was used to be

$$
\sigma_{\mathrm{a}}=\sigma_{\mathrm{f}}{ }^{\prime}\left(2 N_{\mathrm{f}}\right) b
$$

or

$$
\log \sigma_{\mathrm{a}}=\log \sigma_{\mathrm{f}}{ }^{\prime}+b \log 2 N_{\mathrm{f}},
$$

where $\sigma_{\mathrm{f}}$ ' and $b$ are the fatigue strength coefficient and exponent, respectively. The straight line in Fig. 14 was determined by least-square fit. The magnitudes of $\sigma_{\mathrm{f}}{ }^{\prime}$ and $b$ were determined to be $316.43 \mathrm{MPa}$ and -0.12 , respectively.

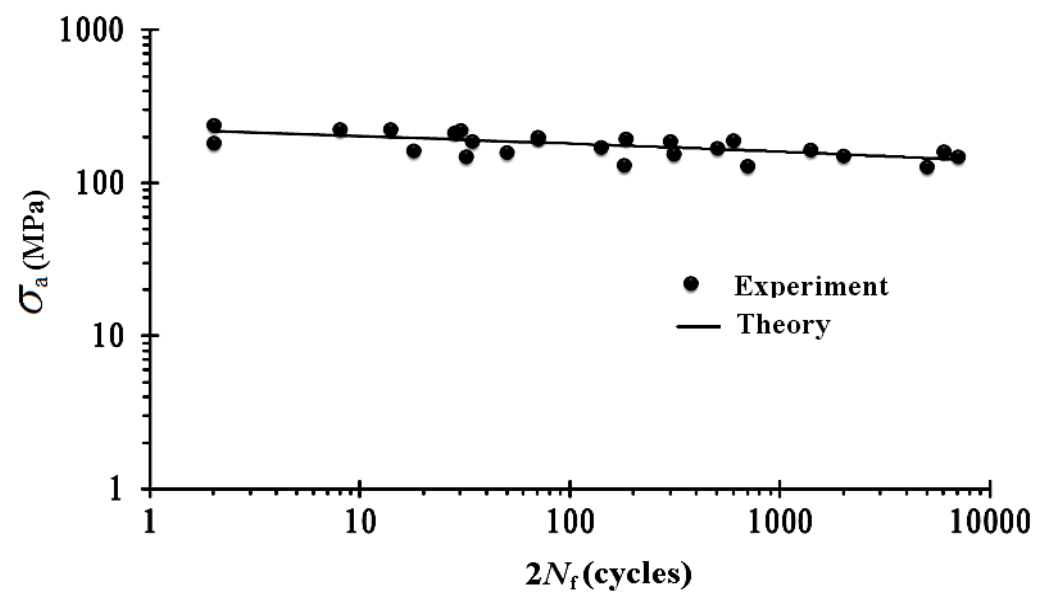

Figure 14: Experimental and simulated stress amplitude $\left(\sigma_{\mathrm{a}}\right)$ versus the number of bending cycles required to produce failure $\left(2 N_{\mathrm{f}}\right)$ for local sharp-dented 6061-T6 aluminum alloy circular tubes with different dent depths under cyclic bending on a log-log scale. 


\section{CONCLUSIONS}

The response and failure of local sharp-dented 6061-T6 aluminum alloy tubes, with different dent depth and submitted to cyclic bending, were investigated. As a result of the experimental and simulated results, some important conclusions have been reached and are presented as follows:

(1) Under symmetrical cyclic bending, the experimental $M-\kappa$ relationship for local sharpdented 6061-T6 aluminum alloy tubes with any $a$ exhibits a closed and stable hysteresis loop. In addition, the sharp dent is small and local, the dent depth has almost no influence on the $M-\kappa$ curve.

(2) Under symmetrical cyclic bending, the experimental $\Delta D_{0} / D_{0}-\kappa$ relationship for local sharp-dented 6061-T6 aluminum alloy tubes with any $a$ exhibits an increase and ratcheting with an increase in the number of bending cycles. The $\Delta D_{0} / D_{0}-\kappa$ curves are symmetrical for $a=0.0 \mathrm{~mm}$ and asymmetrical for $a \neq 0.0 \mathrm{~mm}$. In addition, larger $a$ causes more asymmetry and larger ovalization.

(3) By employing the proper stress-strain relationship, model, mesh, boundary conditions, and loading conditions, ANSYS can describe the behavior of local sharp-dented 6061T6 aluminum alloy circular tubes under cyclic bending. The experimental momentcurvature and ovalization-curvature relationships were compared with the ANSYS simulation. Good agreement between the simulated and experimental results was achieved.

(4) According to the calculated $\sigma_{\mathrm{a}}$ and $N_{\mathrm{f}}$ relationship for local sharp-dented 6061-T6 aluminum alloy circular tubes under cyclic bending. A simple fatigue model (eqn (2)) was used and parameters $\sigma_{\mathrm{f}}{ }^{\prime}$ and $b$ were determined to be $316.43 \mathrm{MPa}$ and -0.12 , respectively. It can be seen that the simulation is in good agreement with the experimental result as shown in Fig. 14.

\section{ACKNOWLEDGEMENT}

The research was executed with the support of the Ministry of Science and Technology under grant MOST 103-2221-E-006-041. We sincerely thank them for their assistance.

\section{REFERENCES}

[1] Pan, W.F., Wang, T.R. \& Hsu, C.M., A curvature-ovalization measurement apparatus for circular tubes under cyclic bending. Experimental Mechanics, 38(2), pp. 99-102, 1998.

[2] Pan, W.F. \& Fan, C.H., An experimental study on the effect of curvature-rate at preloading stage on subsequent creep or relaxation of thin-walled tubes under pure bending. JSME International Journal, Series A, 41(4), pp. 525-531, 1998.

[3] Pan, W.F. \& Her, Y.S., Viscoplastic collapse of thin-walled tubes under cyclic bending. ASME Journal of Engineering Materials and Technology, 120(4), pp. 287-290, 1998.

[4] Lee, K.L., Pan, W.F. \& Kuo, J.N., The influence of the diameter-to-thickness ratio on the stability of circular tubes under cyclic bending. International Journal of Solids and Structures, 38(14), pp. 2401-2413, 2001.

[5] Lee, K.L., Pan, W.F. \& Hsu, C.M., Experimental and theoretical evaluations of the effect between diameter-to-thickness ratio and curvature-rate on the stability of circular tubes under cyclic bending. JSME International Journal, Series A, 47(2), pp. 212-222, 2004.

[6] Chang, K.H. \& Pan, W.F., Buckling life estimation of circular tubes under cyclic bending. International Journal of Solids and Structures, 46(2), pp. 254-270, 2009. 
[7] Lee, K.L., Hung, C.Y. \& Pan, W.F., Variation of ovalization for sharp-notched circular tubes under cyclic bending. Journal of Mechanics, 26(3), pp. 403-411, 2010.

[8] Lee, K.L., Mechanical behavior and buckling failure of sharp-notched circular tubes under cyclic bending. Structural Engineering and Mechanics, 34(3), pp. 367-376, 2010.

[9] Lee, K.L., Hsu, C.M. \& Pan, W.F., Viscoplastic collapse of sharp-notched circular tubes under cyclic bending. Acta Mechanica Solida Sinica, 26(6), pp. 629-641, 2013.

[10] Lee, K.L., Hsu, C.M. \& Pan, W.F., Response of sharp-notched circular tubes under bending creep and relaxation. Mechanical Engineering Journal, 1(2), pp. 1-14, 2014.

[11] Limam, A., Lee, L.H. \& Kyriakides, S., On the collapse of dented tubes under combined bending and internal pressure. International Journal of Solids and Structures, 55(1), pp. 1-12, 2012. 